European Association for the
and Power Quality (EA4EPQ)

International Conference on Renewable Energies and Power Quality (ICREPQ'12)

Santiago de Compostela (Spain), 28th to 30th March, 2012

\title{
CFD simulations for investigating the wake states of a new class of tidal turbine
}

\author{
Mulualem G. Gebreslassie, Gavin R. Tabor, Michael R. Belmont \\ College of Engineering, Mathematics and Physical Sciences \\ Harrison building, Streatham Campus \\ University of Exeter \\ North Park Road, Exeter, UK, EX4 4QF \\ Phone: +447550747812, E-mail: mgg204@exeter.ac.uk; \\ G.R.Tabor@exeter.ac.uk; M.R.Belmont@exeter.ac.uk
}

\begin{abstract}
The interest of tidal energy exploitation is increasing in recent years promoting the need for development of efficient tidal turbines. This paper investigates the wake states of energy extraction by a new tidal turbine design, the Momentum Reversal Lift (MRL), developed by Aquascientific Ltd with a different method of tidal turbine modelling (body force) using an open source computational fluid dynamics (CFD) code, OpenFOAM.

The body force CFD model results showed better turbulent patterns downstream of the turbine showing its quality compared to porous disc method which has been used for tidal turbine modelling. The simulations on a single MRL device showed that the wake recovers into $94 \%$ of the free stream at $20 \mathrm{D}$ downstream of the turbine. The performance of a downstream turbine simulated with 20D spacing was reduced by almost $7.17 \%$ due to the energy shadowing created by the upstream wake. Thus, it is necessary to have at least 20D turbine spacing to maintain more than $92 \%$ of the performance of an isolated turbine.
\end{abstract}

Keywords: MRL; Wake; Body Force; LES; CFD

\section{Introduction}

The growing of worldwide energy demand, together with the unsustainable source of conventional energy led to considerable interest in renewable energy development over many years. Tidal energy is one of the renewable energies emerging as a possible source for some countries situated in a better site.

A wide range of tidal stream device designs are currently under development and testing with the aim of improving their efficiencies and understanding the flow descriptions in and around the turbine region. A new tidal turbine design (Fig. 1), the Momentum Reversal Lift (MRL), developed by Aquascientific Ltd is the focus of this study.

The study of wake effects of energy extraction by tidal turbine is crucial because of its importance for understanding the flow conditions within and around the turbine region, and the length scale where the turbulence patterns sustain downstream. Several studies have been carried out on the conventional tidal stream turbines using porous disc modelling method to understand the flow conditions. A comparison of both CFD and experimental wake effects by [1] using $10 \mathrm{~cm}$ disc showed that the wake recovered to $90 \%$ of the free stream at 20D downstream, but the experimental results showed faster recovery compared to the CFD modelling. A CFD study by [2] and Laboratory investigation by [3] using a small scale horizontal axis turbine of $40 \mathrm{~cm}$ in diameter showed a free surface deformation immediately downstream of the device with the CFD simulation confirming similar findings.

This paper presents the result of CFD simulations of wake states modelled using a different turbine modelling method (body force) for creating momentum change on the fluid flow by the actual dimensions of the MRL turbine blades. The aim being to gain an understanding of the flow descriptions around the turbine during the extraction of tidal energy by the MRL device. The main interests are the turbulent patterns and the length scale it can sustain downstream of the turbine and the effect of the energy extraction on the free surface. Moreover, the paper contains a study of the energy shadowing between an array of two MRL devices.

\section{MRL turbine}

Aquascientific Ltd developed a new tidal turbine (shown in Fig. 1) to get a better performance compared with the conventional tidal turbines because of its suitability to use near the free surface and its design of stretching across a channel (high aspect ratio) which enables to use the blockage effects. This device is a horizontal axis but operates differently to the conventional horizontal axis turbines.

The turbine is constructed with three blades, which rotate about their own axis and mutually central axis (shaft axis), creating a relative rotation between the blades and the shaft. The nominal rotation of the blade is half of that of the central axis to achieve consistent, positive torques from the lift and drag forces [4] although it is still under investigation and could be subjected to some changes. The axis 
of rotation is perpendicular to the flow direction in contrast to the conventional horizontal axis turbines where the flow direction is along the axis of rotation.

The torque contribution by the lift and drag force is positive over the whole cycle allowing to produce large torque as described by [4]. The MRL turbine is under testing with a couple of different size prototypes. Experimental study showed that its efficiency could reach up to $50 \%$ as explained by [4].

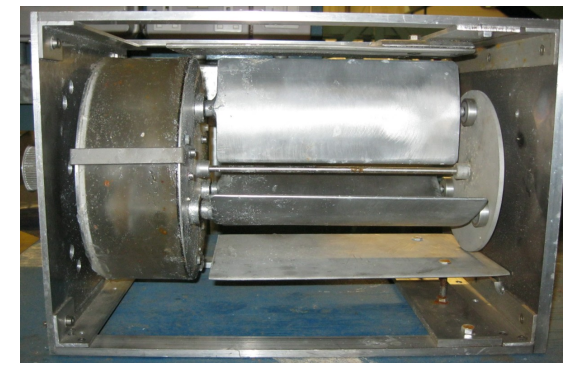

Figure 1: MRL Turbine

\section{Methodology}

\section{A. Computational modelling}

Direct numerical simulation (DNS) is the most accurate approach for complex turbulence simulations by solving the unsteady Navier-stokes equations [5]. DNS resolves all the motions and requires exceptionally fine meshing making it computationally extremely expensive.

Researchers have been developing alternative techniques for reducing the computational cost, but balancing the satisfaction of the engineering solutions of turbulent motions. Reynolds-averaged Navierstokes (RANS) and Large Eddy Simulations (LES) techniques are common modelling methods developed and used as an option for solving turbulent motions. RANS models all the motions and is computationally fast and inexpensive compared to the DNS. However, it is deficient in describing the turbulent motions due to its averaging and simplifications of modelling.

LES modelling approach resolves the large-scale turbulent motions by filtering the Navier-stokes equations and the small-scale motions are modelled using sub-grid scale (SGS) methods. LES is a compromise between DNS and RANS. A simulation of the MRL turbine was carried out using the RANS and LES models. However, the results from the LES gave a better reproduction of turbulent patterns making it a logical choice for this study.

\section{B. LES governing equations}

The LES governing equation used for the simulation is a combination of the filtered NS equations and source terms as shown in Eqn. (2). The first two source terms ( $\rho g$ and $F_{s}$ ) were added to the existing NS equation for solving two incompressible fluids capturing the interface using a volume of fluid (VOF) method [6]. In this study, a new source term, body force $\left(F_{b}\right)$, was added to the existing LES model to create a momentum change in the fluid flow by the turbine blades. The complete continuity and momentum equation of the LES is then given as:

Continuity equation:

$$
\nabla \cdot U=0
$$

Momentum equation:

$$
\underbrace{\frac{\partial \rho \rho}{\partial t}+\nabla \cdot(\rho U U)=-\nabla p+\nabla \cdot \mu \nabla U}_{\text {Filtered NS equations }}+\overbrace{\rho g+F_{s}+F_{b}}^{\text {Source terms }}
$$

Where: $\mathrm{U}$ is the velocity, $\rho$ is the density, $\mathrm{p}$ is the pressure, $\mu$ is the dynamic viscosity, $\mathrm{g}$ is the gravitational acceleration, $F_{s}$ is the surface tension, and $F_{b}$ is the body force.

The filtered Navier-stokes equations (continuity and momentum equations) are used simultaneously for describing the flow of the two phases. The physical properties of one fluid is calculated as weighted averages based on the volume fraction of the two fluids in one cell.

\section{1) Volume of fluid method}

The MRL turbine need to be simulated in a channel with a sharp interface or free surface on the top, which requires determining of the relative volume fraction of the two phases in a computational cell. VOF method has been widely used in the study of floating body applications, breaking waves, non linear free surface flows and other multiphase flows to handle those kinds of problems as documented by $[7,8,9,10]$. It is an easy, flexible and efficient method for treating free boundaries as described by [11] and was used in this study coupled with LES model.

The volume of fluid in a cell is calculated as $F_{v o l}=\alpha V_{\text {cell }}$, where $V_{\text {cell }}$ is the volume of a computational cell and $\alpha$ is the fluid fraction in a cell. If the cell is filled with fluid then $\alpha=1$ and if it is void, its value should be 0 . At the interface between the two phases, the value of $\alpha$ is between 0 and 1 . The value of $\alpha$ is calculated from a separate transport equation as:

$$
\frac{\partial \alpha}{\partial t}+\nabla \cdot(\alpha U)=0
$$

During the simulations, there is a surface compression and in OpenFOAM, an extra artificial compression term which is active only on the interface region is introduced into eqn. (3) as follows:

$$
\frac{\partial \alpha}{\partial t}+\nabla \cdot(\alpha U)+\nabla \cdot\left(\alpha(1-\alpha) U_{r}\right)=0
$$

Where: $U_{r}$ is a velocity field suitable to compress the interface. The physical properties $(\mu$ and $\rho$ ) at any point in the domains are calculated as a weighted averaged of the volume fraction of the two fluids, $\alpha$, as:

$$
\begin{gathered}
\mu=\alpha \mu_{f}+(1-\alpha) \mu_{g} \\
\rho=\alpha \rho_{f}+(1-\alpha) \rho_{g}
\end{gathered}
$$

Where: the subscripts $(f$ and $g$ ) on the physical properties represents the two phases.

\section{2) Surface tension}

A momentum term due to surface tension developed by [12] is added in the Navier-stokes momentum equation to take into account the surface tension effects on the fluid flow due to discontinuous change of fluid properties. This surface tension can be computed as:

$$
F_{s}=\sigma \kappa(x) \mathbf{n}
$$


Where $\mathbf{n}$ is a unit vector normal to the interface and it can be calculated from the fluid fraction as:

$$
\mathbf{n}=\frac{\nabla \alpha}{|\nabla \alpha|}
$$

The curvature of the interface $(\kappa(x))$ can be computed as:

$$
\kappa(x)=\nabla \cdot \mathbf{n}
$$

\section{3) Turbine modelling}

The principle of actuator disc frequently used for modelling turbines both in experimental and computational studies with a porous disc method. The disc acts as a momentum sink reducing the flow momentum which matched to the thrust coefficients of the turbine [13].

Several studies have been carried out using the porous disc method such as simulation of two dimensional flow with a free surface by [14], simulation of the horizontal axis turbine and comparison of the far wake with experimental data [1,15], and for the study of proximity [13]. Studies by [1, 2, 13] showed that the porous disc method minimizes the requirement of mesh refinement and or modelling the geometry in full, reducing the associated computational costs and has shown reasonable agreement with experimental data, but [1] acknowledged that the vortex shedding from the edge of the disc is not similar to the real turbines. The study by [13] also showed that the porous disc method has no capability of resolving the flow around each blade except reducing the momentum of the fluid as it passes through the disc.

The porous disc method was used to simulate the MRL turbine but fails to reproduce a complex motion within the turbine region and turbulence patterns downstream of the turbine. Thus, an appropriate method has to be developed to overcome the pitfalls of the porous disc method, but the simplicity should be maintained to minimise the computational power. This paper introduced a different tidal turbine modelling using a body force applied by each of the blades creating both a momentum change and rotational flow. The body force can be written as:

$$
F_{b}=F_{D}+F_{L}
$$

The code was developed considering drag and lift forces applied by the blades on the fluid flow as shown in Fig. 2. An extra body forces were imposed to the annular section, the portion where the whole turbine rotates as shown in Fig. 2 to represent the rotational effect of the whole turbine on the fluid flow. The addition of the forces in the annular section produces a circulation of fluid on the circumferential of the turbine region similar to the actual turbines does.

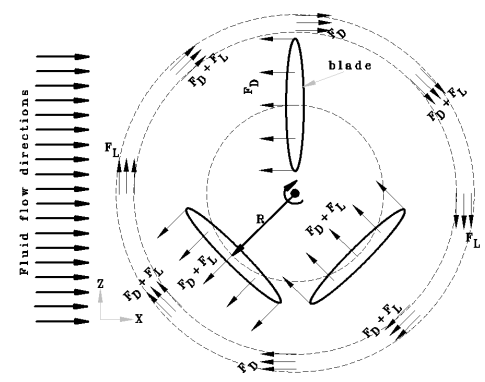

Figure 2: Body forces applied by each blade

The body force was estimated based on a torque measured during experimental study of the performance of the small scale MRL turbine given in Fig. 1.

\section{Sub-grid scale model}

The sub-grid scale (SGS) stress resulted from the filtering processes of the Navier-stokes equations are unknown and needs modelling. The stress is a large scale momentum flux resulted by the unresolved scales. Usually, those stresses are approximated by SGS models developed based on the eddy viscosity due to their difficulties. The most popular SGS models extensively used for simulations include One equation eddy viscosity, Smagorinsky, the kinetic-energy model and the dynamic model. The most commonly employed hypothesis in the SGS turbulence models is the Boussinesq hypothesis [16], which calculates the SGS stress using a linear relationship with the rate of strain tensor.

The earliest SGS model is the Smagorinsky model proposed by [17]. Though the Smagorinsky model has been relatively successful, it has had some problems especially if the flow is extremely complex and has an internal shear flows. Several modifications have to be made if used for channel flow simulations as described by [5] and so a better model has to be chosen for the MRL simulations.

The one-equation eddy viscosity model (oneEqEddy) developed by [18] has been used in a wide range of turbulent problems and is popular as described by [19] because of its reasonable results. Thus, the one-equation model was used to model the small-scale motions in the MRL turbine simulation.

\section{Computational set up}

\section{1) Computational domain geometries}

Fig. 3 is a schematic representation of 3D geometry used as a computational domain and comprises the MRL turbine blade dimensions.

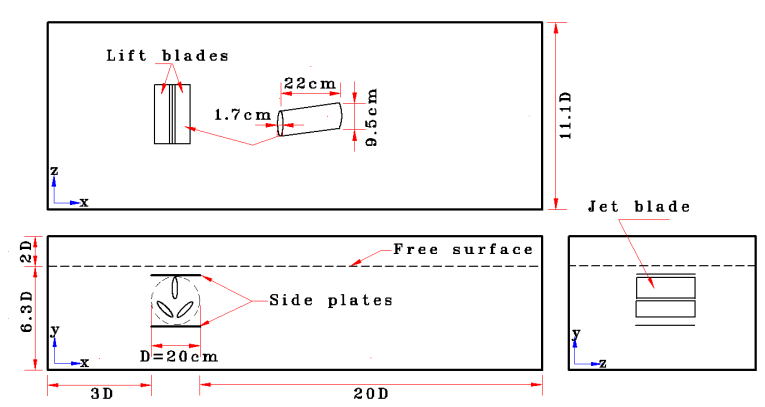

Figure 3: Computational domain geometry

The overall diameter of the MRL turbine shown in Fig. 1 is $\mathrm{D}=$ $0.20 \mathrm{~m}$ and the dimensions of the computational domain are given based on the diameter as shown in Fig. 3. The computational domain is occupied by two phases, water at a depth of $6.3 \mathrm{D}$ and the rest occupied by air before the computation starts.

The number of cells used in the computational domain is in the order of 149040. The cell sizes were refined around the turbine region for creating uniform computation because of its zone of interest. 


\section{2) Implementation of boundary condition}

The computational domain contains seven boundary patches namely atmosphere, seaBed, wall, water and air inlet and outlet. The top part of the domain is an atmosphere which represents a standard patch. This top boundary is free to the atmosphere and allows both outflow and inflow according to the internal flow. This was developed by a combination of pressure and velocity boundary conditions [6].

seaBed and wall are the floor and the front and back side of the computational domain respectively, and both patches represent wall. zeroGradient boundary condition was applied to the pressure field.

Inlet represents the patch where the water phase enters to the domain. At the inlet, a parabolic velocity field is applied to account the difference of the fluid flow along the depth of the channel. This velocity field have been studied for many years, and it is proved by $[20,21,22,23]$ that the maximum velocity occurs slightly below the free surface because of the resistance at the interface between the two phases, water and air. [20] had specifically described that the maximum velocity occurs at one third of the water depth from the free surface.

A parabolic velocity profile was developed by [6] for a calculation of fluid flow in a tube, and the code was modified to match a parabolic inlet velocity profile for a channel flow based on the location of the maximum velocity given by [20] and imposed in to the inlet patch of the computational domain. The span-wise velocity profile showed nearly constant except a small falls near the wall boundaries.

\section{Results and discussions}

\section{A. Effect of turbine submerging relative to the free surface}

The MRL device is designed to operate near a free surface flow which brings the issue of free surface effect. Therefore, a simulation was carried out with the turbine submerged to get the appropriate location for the device to be placed. The velocity across the depth of the domain is different as described in the previous section, but it was assumed uniform value to get constant kinetic energy for this simulation to minimise its effect on the comparison of turbine submerging. Otherwise, if the inlet velocity is considered as parabolic, the different kinetic energy available on the depth of the domain will have its own effect, making it difficult for the comparison of the effect of energy extraction by the turbine on the free surface.

It seems difficult to visualise the difference between Figs. $4 \mathrm{a}$ and $4 \mathrm{~b}$, but the effect of energy extraction on the free surface decreases as the turbine moved deeper from the free surface as shown in Fig. $4 \mathrm{~b}$. However, the ground may have some effect on the performance of the turbines if moved closer to it though there is no interest to examine this effect for now as the turbines are designed to operate near a free surface flow. In contrast, as the turbine moved closer to the free surface, the surface deformation increases as shown in Fig. 4a.

The effects on the free surface shown in Fig. 4 can be clearly visualised using the phase fraction at the interface of the two phases. Fig. 5 shows that the free surface increases upstream of the turbine, which is above the initial phase fraction of 0.5 , due to the blockage effect and drops immediately downstream of the turbine for both positions $(0.25 \mathrm{D}$ and $1 \mathrm{D})$ and recovers farther downstream into its initial state. It is understood that as the turbine moves closer to the surface $(0.25 \mathrm{D})$, that turbine has more influence on the free surface deformation compared to the turbine deeper in the domain (1D) as shown in Fig. 5.

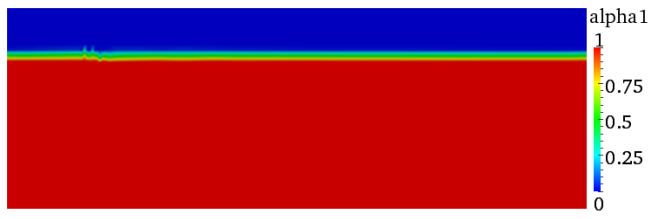

(a) With plate at depth of $0.25 \mathrm{D}$

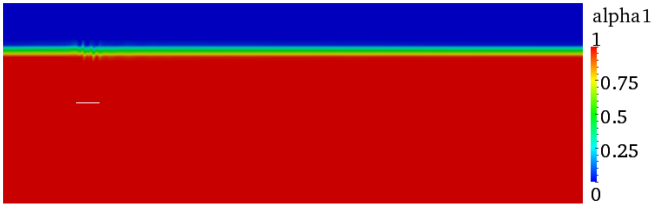

(b) with plate at depth of $1 \mathrm{D}$

Figure 4: Free surface deformation at different positions of the device relative to the free surface

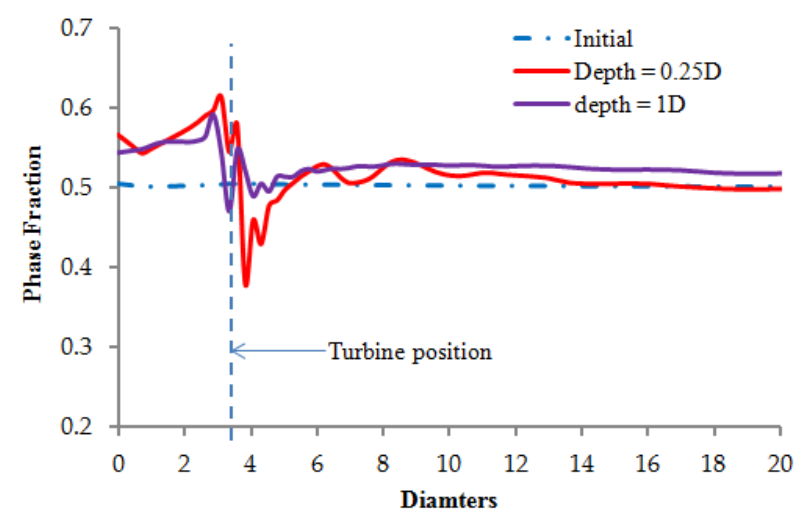

Figure 5: Phase fraction changes of the free surface at different depths of turbine position

The velocity deficit along the centreline through the turbine is barely affected by the turbine positions at 0.25 and 1 diameters depth (from the top of the plate to the free surface) as shown in Fig. 6. This is an indication that the energy extraction may not be affected by the position of the turbines if the velocity across the depth of the domain is equal.

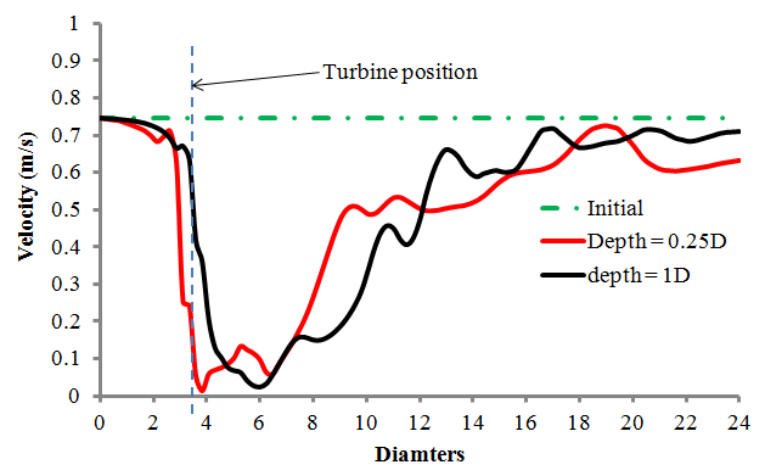

Figure 6: Centreline stream-wise velocity deficit at different depth of turbine position 


\section{B. Velocity Deficit at different cross-sections}

The velocity profile at the inlet (upstream $=0 \mathrm{D}$ ) was used as a base for comparison of velocity profiles at five different cross-sections of the domain.

The velocity deficit increases as the fluid passes through the turbine and recovers back farther downstream as shown in Fig. 7 and 8. The flow becomes complex within the turbine region as expected due to the complexity of the drag and lift forces applied on the fluid flow. The velocity profile at the end of the domain shown in Fig. 8 (downstream $=20 \mathrm{D}$ ) recovers into more than $94 \%$ of the stream flow (upstream $=$ 0D).

The velocity profiles slightly below and above $1.35 \mathrm{~m}$ in the air body (Fig. 8) have no equivalent evolution comparing to the base velocity profile in which the air body may have been affected by the wave created at the sharp interface between the air and water as the water flows along the domain.

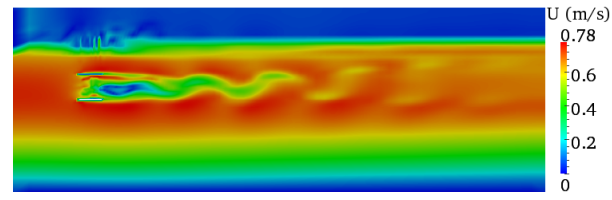

(a) Side view

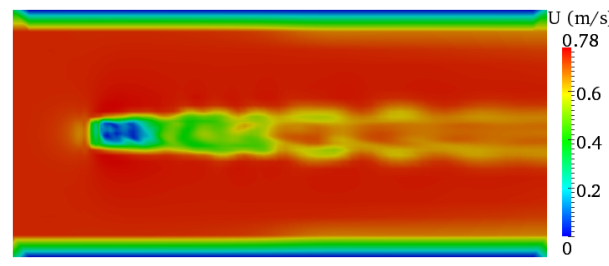

(b) Top view

Figure 7: Velocity contours along the centreline plane of the turbine at $\mathrm{t}=200 \mathrm{~s}$

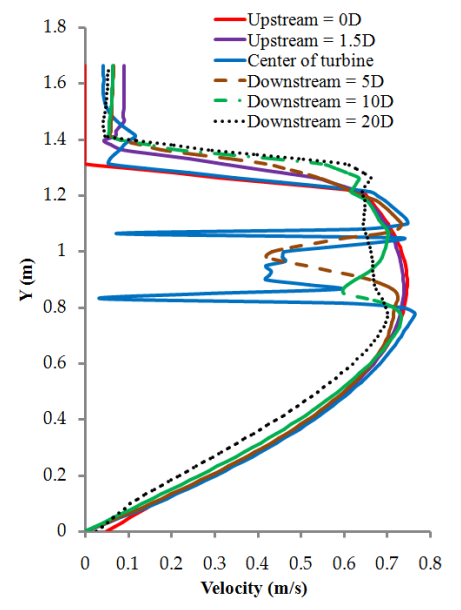

Figure 8: Vertical velocity profiles at different cross-sections for the velocity contours given in Fig. 7

\section{Energy shadowing of an array of MRL devices}

A tidal stream farm could have several turbine location configurations. A detailed study of the influence of the proximity of those turbines is crucial for understanding the energy shadowing, and the flow conditions of the farm. One of the Configurations we might get in a farm is a row of turbines located along the direction of the flow.

To examine the turbine to turbine interaction, two MRL turbines configured with 15D and 20D turbine spacing were used for the simulations in this study. Fig. 9 shows the velocity contours for the two configurations. The wake interaction is larger in the $15 \mathrm{D}$ turbine spacing configuration which can be seen in Fig. 9a and more clearly on the XZ plane given in Fig. 9b. As the spacing between the turbine increases to 20D, the wake interaction decreases as shown in Figs. 9c and $9 \mathrm{~d}$.

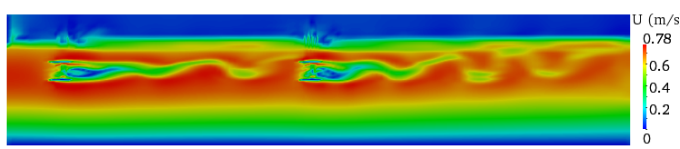

(a) XY plane $15 \mathrm{D}$ spacing

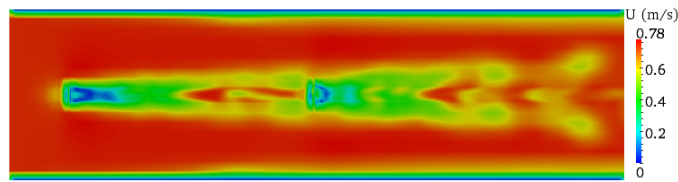

(b) XZ plane $15 \mathrm{D}$ spacing

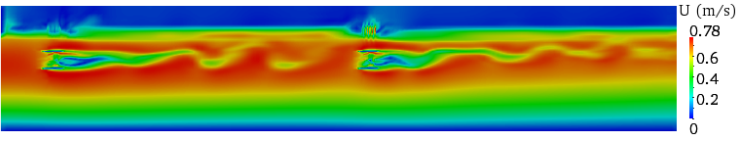

(c) XY plane 20D spacing

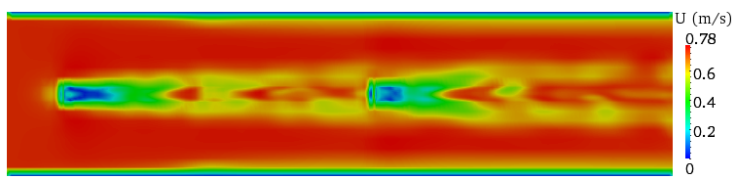

(d) XZ plane 20D spacing

Figure 9: Velocity contours of two turbines at different turbine spacing

Fig. 10 shows the velocity deficit through the centerline along the stream direction for the two configurations (15D and 20D) and the result showed that the upstream turbine affects the downstream turbine resulting a lower turbine velocity when the turbines were placed at $15 \mathrm{D}$ compared to the turbine at $20 \mathrm{D}$ turbine spacing.

To get a clear understanding of the influence of upstream turbines, the results from the two configurations was compared to the simulation result of a base case (isolated turbine). The velocity deficit immediately downstream of the turbine is lower than the base case velocity deficit as shown in Fig. 11a between 20D and 22D which is a result of the upstream wake interaction with the downstream turbine. The given turbine spacing (15D) is not enough for recovering the wake created by the upstream turbine before reaching the downstream turbine.

The result was improved by increasing the turbine spacing into 20D. The velocity deficit both for the base case and the downstream 


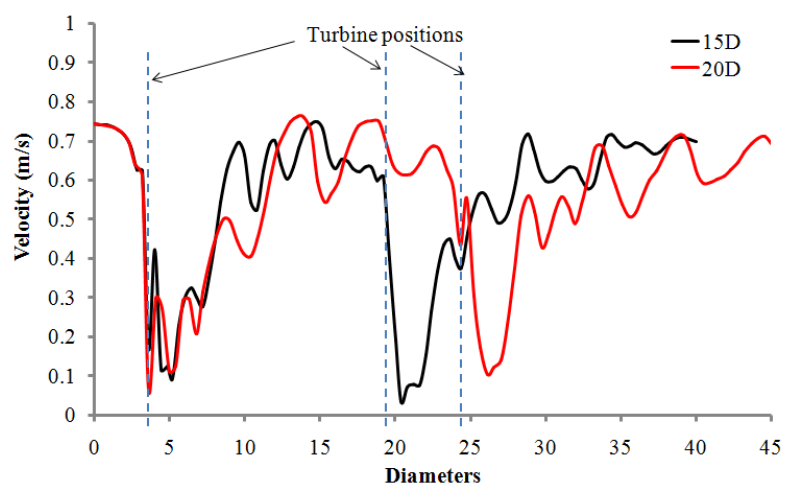

Figure 10: Centreline velocity gradient through the two turbines for the two configurations (15D and 20D)

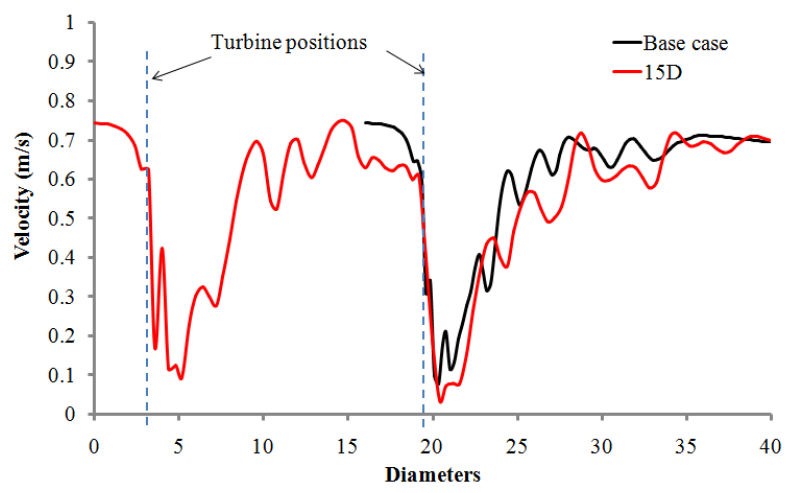

(a) $15 \mathrm{D}$ turbine spacing

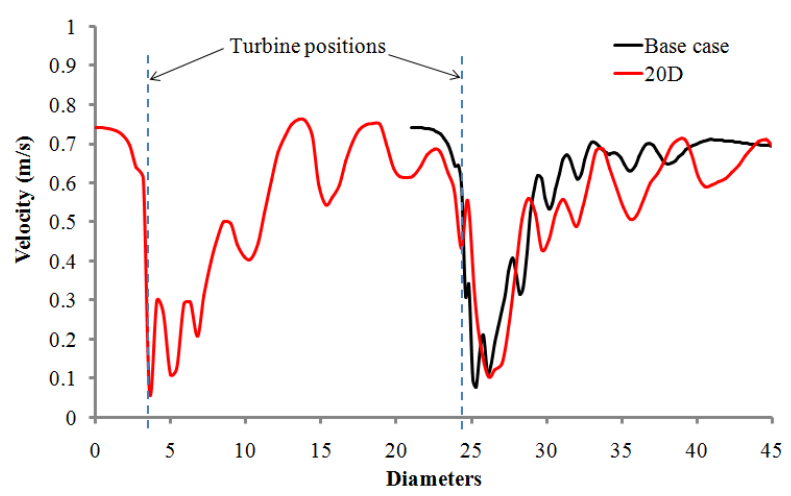

(b) 20D turbine spacing

Figure 11: Comparison of the centreline velocity gradient through the two turbines and an isolated turbine

turbine is almost similar, which is an indication that a 20D turbine spacing between upstream and downstream turbine might be enough to get a reasonable performance by isolated MRL turbines.

So far, the velocity was used to investigate the energy shadowing between the two turbines, but the result can be analysed using the pressure drops across the turbine due to the change in momentum on the flow. Fig. 12 show the pressure gradient line along the stream flow through the centreline of the turbine, and it was compared with the pressure gradient line of the base case. The pressure drop by the upstream turbine shown in Fig. 12a is larger than the pressure drop by the same turbine shown in Fig. $12 \mathrm{~b}$ and by the downstream turbine in both figures. The possible reason is that due to the turbine to turbine spacing between the upstream and downstream, the small the turbine spacing affects the pressure on the upstream turbine more severely due to the blockage effect and led to produce a larger pressure drop compared to turbines which have larger spacing.

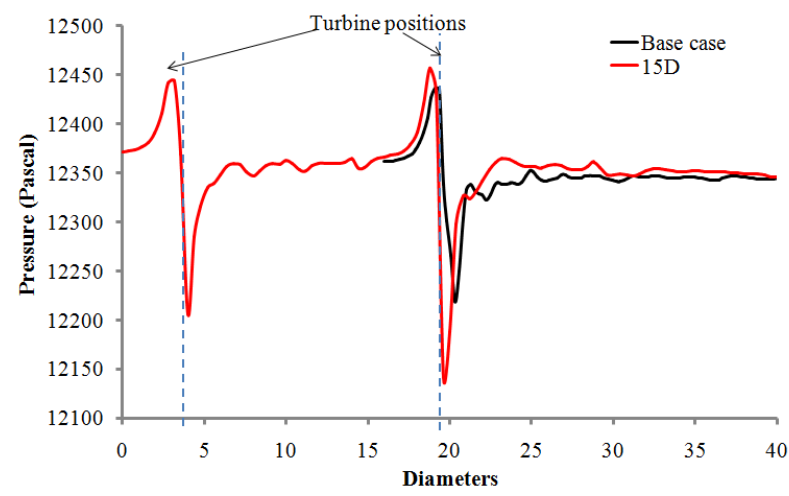

(a) $15 \mathrm{D}$ turbine spacing

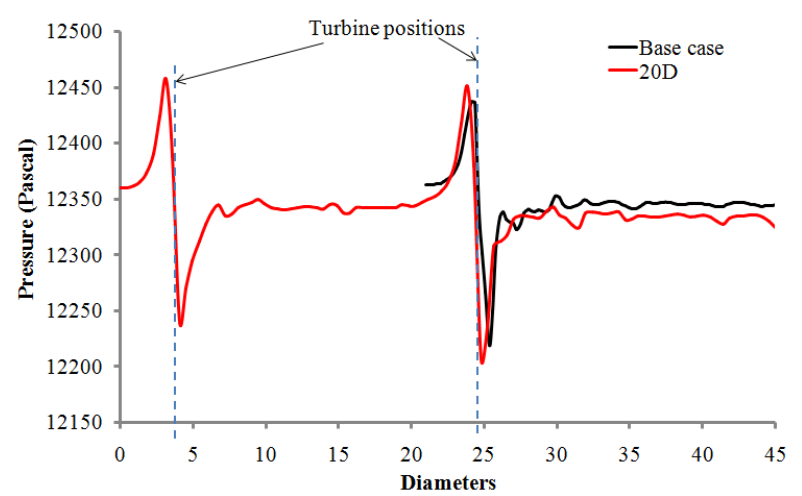

(b) 20D turbine spacing

Figure 12: Comparison of the centreline pressure gradient through the two turbines and an isolated turbine

There is a high pressure drop on the downstream turbine in both configurations as shown in Fig. 12. However, the pressure drop across the downstream turbine with the $15 \mathrm{D}$ turbine spacing configuration (Fig. 12a) is larger than the pressure drop across the downstream turbine in the 20D spacing (Fig. 12b). The main reason is that the turbine to turbine interaction with the turbines spaced 15D apart led to reduce the kinetic energy of the flow approaching the downstream turbine. This led to unbalanced momentum of the fluid and the applied body force consequently increased the pressure drop.

A more detailed study were carried out using the power extracted by both turbines. The power extracted from the stream flow by the upstream turbines in both configurations were expected to be similar to the base case. However, the results showed a different value which could be affected due to the presence of downstream turbines.

The power extraction by the downstream turbine in the first configuration (15D spacing) was seriously affected and reduced its performance by as much as $18.53 \%$ compared to the same turbine in the second configuration (20D spacing) which was reduced its performance by $7.17 \%$. The performance of both of the turbines in the two configurations were compared with the same but isolated turbine (base case). The reason is mainly due to the upstream turbine wake interaction with the downstream turbine. Therefore,the turbines should be 
spaced 20D apart to maintain more than $92 \%$ of the power extraction performance of an isolated turbine.

\section{Conclusion}

The results of the CFD model showed much of the expected flow behaviours such as the turbulence patterns and the free surface deformation, which was affected by the extraction of energy from the free stream flow using the MRL device. This is an indication of the capability of the CFD model for describing the flows within and around tidal turbines.

The simulations on a single MRL device showed that the wake recovers into $94 \%$ of the free stream at 20D downstream of the turbine. The simulation results from the two configurations showed that the turbine with $15 \mathrm{D}$ spacing inflicted high energy shadowing and reduced the performance of the downstream turbine by around $18.53 \%$. However, the turbine simulated with 20D spacing was reduced its performance by $7.17 \%$. Thus, it is necessary to have at least 20D turbine spacing to maintain more than $92 \%$ of the performance of an isolated turbine.

\section{Acknowledgement}

The study was financially supported by University of Exeter and the authors would like to thank the University for the fund.

\section{References}

[1] ME Harrison, WMJ Batten, LE Myers, and AS Bahaj. Comparison between cfd simulations and experiments for predicting the far wake of horizontal axis tidal turbines. Renewable Power Generation, IET, 4(6):613-627, 2010.

[2] X. Sun, JP Chick, and IG Bryden. Laboratory-scale simulation of energy extraction from tidal currents. Renewable Energy, 33(6):1267-1274, 2008.

[3] L. Myers and AS Bahaj. Wake studies of a 1/30th scale horizontal axis marine current turbine. Ocean Engineering, 34(56):758-762, 2007.

[4] A.P. Janssen and M.R. Belmont. Initial research phase of MRL turbine. Technical report, Technical Report N0:MO 562L, 2009.

[5] J.H. Ferziger and M. Perić. Computational methods for fluid dynamics, volume 2. Springer Berlin, 1999.

[6] OpenCFD Ltd. User guide. http: / / www . open foam. com/ docs/user/, 2011.

[7] C. Yang, R. LOHNER, and H. Lu. An unstructured-grid based volume-of-fluid method for extreme wave and freelyfloating structure interactions. Journal of Hydrodynamics, Ser. $B, 18(3): 415-422,2006$.

[8] C. Yang, R. Lohner, and S.C. Yim. Development of a cfd simulation method for extreme wave and structure interactions. In Proceeding of 24th Int. Conference on Offshore Mech. and Arctic Eng.(OMAE2005), Halkidiki, Greece, pages 12-17, 2005.
[9] G. Chen, C. Kharif, S. Zaleski, and J. Li. Two-dimensional navier-stokes simulation of breaking waves. Physics of fluids, 11:121, 1999.

[10] X. He, S. Chen, and R. Zhang. A lattice boltzmann scheme for incompressible multiphase flow and its application in simulation of rayleigh-taylor instability* 1 . Journal of Computational Physics, 152(2):642-663, 1999.

[11] C.W. Hirt and B.D. Nichols. Volume of fluid (vof) method for the dynamics of free boundaries* 1 . Journal of computational physics, 39(1):201-225, 1981.

[12] JU Brackbill, D.B. Kothe, and C. Zemach. A continuum method for modeling surface tension* 1 . Journal of computational physics, 100(2):335-354, 1992.

[13] S. Gant and T. Stallard. Modelling a tidal turbine in unsteady flow. In Proceedings of the Eighteenth (2008) International Offshore and Polar Engineering Conference, pages 473-479, 2008.

[14] S. Draper, GT Houlsby, MLG Oldfield, and AGL Borthwick. Modelling tidal energy extraction in a depth-averaged coastal domain. Renewable Power Generation, IET, 4(6):545-554, 2010.

[15] LE Myers and AS Bahaj. Experimental analysis of the flow field around horizontal axis tidal turbines by use of scale mesh disk rotor simulators. Ocean Engineering, 37(2-3):218-227, 2010.

[16] J. Boussinesq. Théorie de l'écoulement tourbillant (theories of swirling flow), mém. prés. par div. savants à l'acad. Sci. Paris, $23,1877$.

[17] J. Smagorinsky. General circulation experiments with the primitive equations. Monthly weather review, 91(3):99-164, 1963.

[18] A. Yoshizawa and K. Horiuti. A statistically-derived subgridscale kinetic energy model for the large-eddy simulation of turbulent flows. Journal of the Physical Society of Japan, 54(8):2834$2839,1985$.

[19] S. Deck, P. Duveau, P. d'Espiney, and P. Guillen. Development and application of spalart-allmaras one equation turbulence model to three-dimensional supersonic complex configurations. Aerospace science and technology, 6(3):171-183, 2002.

[20] L. Gordon. Mississippi river discharge. RD Instruments, San Diego, Calif, 1992.

[21] S.Q. Yang, S.K. Tan, and S.Y. Lim. Velocity distribution and dipphenomenon in smooth uniform open channel flows. Journal of hydraulic engineering, 130:1179, 2004.

[22] M.H. Chaudhry. Open-channel flow. Springer Verlag, 2008.

[23] C.L. Chiu and N.C. Tung. Maximum velocity and regularities in open-channel flow. Journal of hydraulic engineering, 128(4):390-398, 2002. 